\title{
Towards Usage Policies for Fuzzy Inference Methodologies for Trust and QoS Assessment
}

\author{
Stefan Schmidt, Robert Steele, Tharam Dillon \\ University of Technology, Sydney, PO Box 123 Broadway \\ NSW 2007 Australia \\ \{sschmidt, rsteele, tharam\} dit.uts.edu.au
}

\begin{abstract}
In this paper, we discuss the benefits of several fuzzy inference system design methodologies and evaluate their characteristics in regard to our trustworthiness and QoS measurement models. Our analysis shows that Mamdani-Assilian or Larsen type and Takagi-Sugeno-Kang type fuzzy inference methods have their merits in different situations. We propose to equip an autonomous agent which acts on behalf of a human being with a policy table enabling the agent to dynamically decide which fuzzy inference system it will select during the trustworthiness evaluation process. We argue that in most situations the Mamdani-Assilian or Larsen type fuzzy inference system represents the preferred choice. However, in situations where the fuzzy rulebase is large, the Takagi-Sugeno-Kang type fuzzy inference system should be chosen due to its superior performance characteristics. This way the agent can perform its tasks more efficiently by choosing the appropriate calculation method depending on the given circumstances.
\end{abstract}

The assessment of trust and credibility is part of our daily life - it happens subconsciously and is based on recommendations, past experiences and vague feelings. Reliable and precise measurement of trust and credibility is especially important if we want to achieve autonomous interactions of intelligent agents in unsupervised distributed environments. However, the replication of such social behavior in information systems represents a major challenge.

In an ideal scenario, a person, who wants to purchase goods or consume a service, would instruct his intelligent agent to execute this time-consuming task on his behalf. The agent's duties and responsibilities would involve service discovery, service selection, contract negotiations, service execution or consumption, payments, and reviewing of the delivered service quality.

In previous work, we have proposed models for both, trustworthiness evaluation in distributed environments to support selection of potential services [1], as well as a quality of service (QoS) review model [2]. Both models are based on fuzzy logic [3] which offers a mathematical concept to deal with uncertainty for the calculation of outputs. This ability to offer reasoning capabilities based on uncertain or incomplete information makes it suitable to simulate human reasoning which is based on similar principles. In both models, we have chosen the Mamdani-Assilian (MA) [4] approach for the fuzzy inference process of our models. In separate research, our group has also proposed a fuzzy model based on the Takagi-Sugeno-Kang (TSK) [5] inference method to determine the trustworthiness and credibility of peer agents in distributed environments [6] 
In this paper we discuss the benefits of these and other fuzzy inference system (FIS) design methodologies and evaluate their characteristics in regard to our trustworthiness and QoS measurement models. First, we briefly introduce our fuzzy trust and QoS evaluation models to establish the context for our suitability analysis. Second, we provide details on the fuzzy inference methods before comparing their benefits in the different situations that the agent might encounter. Based on this analysis we will finally introduce a policy based model which assists the agent to select the appropriate FIS for the specific situation the agent encounters.

\section{Related Work}

A number of researchers have proposed models based on fuzzy logic concepts to offer solutions for the computation of trust, credibility, reputation, or QoS. For example, Falcone et al. [7] use Fuzzy Cognitive Maps (FCM) [8] to model the dynamic influence of measured attributes before and during the trust calculation. A different approach is the Regret system [9] which integrates fuzzy concepts into the analysis of social networks in electronic marketplaces. Other fuzzy logic based approaches for the calculation of trust in distributed systems has been developed by [10] and [11]. In previous research, we have also proposed a fuzzy logic based model to offer flexible and efficient approaches for the computation of variables like trustworthiness, credibility, and QoS [2].

Manara et al. [12] have implemented different models in MATLAB to measure the performance between three different fuzzy controllers. They compared their own approach named Conditionally Firing Rules, and the standard approaches MamdaniAssilian, and Takagi-Sugeno-Kang. Their tests show that the Takagi-Sugeno-Kang controller performs faster in most tests compared to the MA controller. However, they limit their comparisons to response time measurements and precision measurements. Youssef [13] compared MA, TSK, Larsen [14], Singleton, and Tsukamoto type fuzzy controllers in a 1-input, 1-output power system relaying system and found the TSK type inference engine preferable due to its computational efficiency resulting in faster response times required for real-time systems.

Most of these fuzzy logic-based models are designed to suit a specific environment or measure a set of model-specific variables. However, none of these papers have so far investigated the suitability of different fuzzy inference methodologies for the tasks of trust and credibility evaluation and QoS measurement. In this paper, we compare the major approaches (MA or Larsen type and TSK type) used for the defuzzification during the inference process and determine their suitability in different situations.

\section{Fuzzy Trust and QoS Assessment}

In this section, we will briefly discuss our model for trust evaluation and QoS measurement to establish the context of our research. 


\section{Fuzzy Trust Evaluation}

In an unsupervised multi-agent environment, the measurement of trust in other agents plays a crucial role during the service selection process where an agent needs to choose between a number of potential business partners which it previously discovered. The selection of a future business partner or service no longer only depends on matching the tangible criteria a service offers but also on the willingness and capability of a potential business partner to deliver quality of service in a given context at a given timeslot. We use the notion trustworthiness, as a measure, to quantify the trust level an agent has in a potential business partner in a given context at a given time slot. Our model describes a trust evaluation process implemented by an agent to measure trust in a future negotiation partner before the negotiation process takes place. In our model, we define the service consumer as Trusting Agent, potential business partners as Recommendation Queried Agents, peer agents who share their opinions about Recommendation Queried Agents as Recommending Agents, and the actually selected business partner as Trusted Agent.

Given that the Trusting Agent has no sufficient information about Recommendation Queried Agents he asks Recommending Agents to deliver their opinions about them within a given context and a given timeslot. These opinions are composed of one or more datasets which contain a trustworthiness value, context, timestamp, and a trustworthiness value range for each business interaction which the Recommending Agent had with the Recommendation Queried Agent in the past. The Trusting Agent creates a weighted average according to the age of the records within a dataset before feeding this value as one of three input variables into its fuzzy inference engine. The second input for the fuzzy inference engine is the agent's credibility value and the third input is the weight of this opinion resulting from the number of records in the dataset which the Recommending Agent delivered

In a next step, these inputs are fuzzified and mapped to the rulebase in order to calculate a crisp output using either the MA or the TSK approach during the fuzzy inference process. After having computed a crisp trustworthiness output value from each opinion delivered, we calculate the average overall trustworthiness values for a particular Recommendation Queried Agent. Furthermore, if the agent holds trustworthiness records about the Recommendation Queried Agent in his individual database, he combines this value with the aggregated trustworthiness value from the delivered opinions using weight factors for his own data and the externally delivered data. Through this aggregation the Trusting Agent finally gains a trustworthiness value for each Recommendation Queried Agent.

These final composite trustworthiness values for each Recommendation Queried Agent are then used as support during the service selection process. The agent representing the selected service is then called Trusted Agent.

\section{Service quality review after a business interaction}

The measurement of the QoS after a business interaction serves several purposes. First and forcmost, the Trusting Agent requires a QoS value to update his trustworthiness value for the Trusting Agent. This trustworthiness value can then be used for 
future trust evaluation processes. It is also useful for providing opinions about the Trusted Agent to peer agents which might ask for this value in future. Second, the QoS value will be used to adjust the credibility value of Recommending Agents which have delivered their opinions on the Trusting Agent within the context of this business interaction. If their opinions were close to the calculated QoS value (within a predefined threshold) then their credibility value will be increased and otherwise decreased. It is noteworthy that our model reduces credibility values to a significantly higher extend if the trustworthiness review calculations have a negative outcome than vice-versa.

The calculation of the QoS value is based on the extended fuzzy logic-based model [2] of CCCI metrics introduced by Chang, Hussain and Dillon [6]. During the contract negotiations between the Trusting Agent and the Trusted Agent a number of quality assessment criteria are defined, communicated and agreed upon by both parties in the contract. After the completion of the business interaction, these criteria are used for the assessment of the contract fulfillment (QoS). The CCCI metrics define three measurement variables for each quality assessment criterion:

Commitment - The commitment variable measures the actual degree of fulfillment of every specified criterion. That is, the commitment to each criterion (service condition), to which the Trusted Agent and the Trusting Agent mutually agreed upon, before the business interaction.

Clarity - The clarity variable provides a measure to establish if each criterion was clearly specified, commonly understood and mutually agreed to between the Trusting and the Trusted Agent.

Influence - The influence variable measures the impact of each criterion on the overall investigated quality as perceived by the agent owner and published in the service contract.

Similar to our fuzzy trust evaluation model, we use a fuzzy inference engine to compute the desired output service quality value for each predefined criterion. We fuzzify the predefined value for influence, the observed value for commitment, as well as the clarity value which can only be determined after the business interaction took place and possible ambiguities in the service contract become clear. The fuzzified inputs for each quality criterion will then be mapped to the predefined rulebase before being inferred in order to gain the service quality value. The two approaches for fuzzy inference are introduced in the following section in greater detail. After having computed service quality values for each quality assessment criterion, we calculate the average overall service quality values for the assessed business interaction.

The overall service quality value represents the correlation between the expected service quality (as defined in the contract) and the actually delivered quality of the product or service. This value can then be used to adjust both, the trustworthiness value for the Trusted Agent as well as the credibility values for the Recommending Agents which supplied their opinions during the trust evaluation process. 


\section{Fuzzy Rules and Inference Methodologies}

In this section, we will discuss and compare the Mamdani-Assilian (MA) or Larsen type and Takagi-Sugeno-Kang (TSK) type fuzzy inference methodologies. First, we must understand the nature of all rules expressed in fuzzy inference systems. Fuzzy rules allow us to characterise imprecise dependencies between the input variables in our trust and QoS assessment models using linguistic variables rather then crisp sets.

The usage of linguistic variables allows the desired freedom for the agent owner to apply his personal understanding and experience to model the system behavior. Furthermore, the design of rules based on linguistic variables is more suitable for agent owners which possess the required knowledge in the domain or context in which the assessment takes place but may not possess sufficient mathematical expertise to encode their knowledge in complex program and data structures. The imprecision involved in the usage of linguistic variables which are represented by overlapping fuzzy sets allow a high level of readability and comprehensibility for human beings.

\section{MA and Larsen Type Fuzzy Inference Systems}

An exemplary MA or Larsen type fuzzy rule which we have used in previous work has the following form [15]:

$$
\text { IF } x_{1} \text { IS } A l^{j} \text { AND } x_{2} \text { IS } A 2^{j} \ldots \text { AND } x_{n} \text { IS } A n^{\prime} \text { THEN } y \text { IS } B j, j=1,2, \ldots, M
$$

where $x_{i}$ for $i=1,2, \ldots, n$ are linguistic input variables, such as credibility or clarity in the trust evaluation model and the QoS assessment model respectively; $A t^{j}$ for $i=1,2, \ldots, n$ are input fuzzy sets such as 'high', 'medium', or 'low'; $y$ is the linguistic output variable such as trustworthiness or service quality; $B j$ is the output fuzzy set, and $M$ is the number of fuzzy rules. Noteworthy for MA and Larsen type inference engines is the fact that the consequent of each rule is also composed of fuzzy sets which are represented as linguistic variables. For each firing fuzzy rule the output of the rule inference (implication) will be mapped to its corresponding output fuzzy set, that is, the result is described in terms of membership in fuzzy sets.

The main difference between Mamdani-Assilian and Larsen type methods lies in the implication of fuzzified variables within a antecedent part of the fuzzy rule and the composition of the resulting membership functions defined by fuzzy variables in the rule consequents. MA proposed a max-min approach which uses the minimum operator for rule implication and the maximum operator for rule composition. Larsen proposed the max-product method which uses the product operator for rule implication and the max operator for rule composition. Apart from those different rule implication and membership function composition methods both approaches have identical characteristics and, therefore, they exhibit a similar performance.

In order to receive the desired crisp output value, a process called defuzzification must be applied. Popular defuzzification approaches include the 'centre of area' method, the 'centre of maxima' method, or the 'mean of maxima' approach [16]. 


\section{Takagi-Sugeno Type Fuzzy Inference}

The newer TSK fuzzy inference approach takes a somewhat different path. While the antecedent block of each fuzzy rule remains the same, the consequent block employs a simple equation which takes the input fuzzy variables into account. This equation can be of linear or quadratic type and is referred to as type-1 or type-2 TSK models [17] respectively. Fuzzy inference systems can also be modeled using fuzzy rules with singleton consequents [18]. However, limited modeling capabilities [17] of singleton type FIS result in more coarse grained results and thus affect the quality of the model.

An example fuzzy rule which represents a type-2 TSK model has the following form:

IF $x_{1}$ IS $A l^{j}$ AND $x_{2}$ IS $A 2^{j} \ldots$ AND $x_{n}$ IS $A n^{\prime}$ THEN $y=f_{j}\left(x_{1}, x_{2}, \ldots, x_{n}\right)$, for $j=1,2, \ldots, M$

The function $f_{\mathrm{j}}$ accomplishes a direct mapping between the fuzzified input variables to the output space $y$. Normally, each of the $r$ rules of the fuzzy system is assigned a weight factor $w$ during the aggregation of all output singletons resulting in the following weighted sum $y_{\text {overall }}$ :

$$
y_{\text {overall }} \frac{\sum_{r}^{i=1} w_{i} y_{i}}{\sum_{r}^{i=1} w_{i}}
$$

The obvious difference to the MA or Larsen Fuzzy inference methods is that the consequent of each rule is not a fuzzy set but instead a singleton.

\section{Comparison between MA or Larsen and TSK Fuzzy Inference}

Now that we briefly discussed the foundation of fuzzy rules and the MA or Larsen type and TSK type fuzzy inference methodologies, we need to compare their respective advantages.

Clearly, the MA or Larsen approaches represent a more intuitive and interpretable approach since the knowledge of the agent owner is applied using linguistic variables rather then linear or quadratic equations which are harder to extract from analyzing human experiences and feelings. However, when using linguistic variables within the consequent block of fuzzy rules it becomes necessary to apply additional calculations in order to generate a crisp output. These additional calculations (defuzzification) require more computational resources and can thus result in slower system performance compared to TSK inference systems. This is especially the case if the fuzzy inference engine is used for a large number of calculations or contains a large set of rules. Furthermore, the MA approach offers the flexibility to choose the defuzzification method which the agent owner finds most suitable in a given context. However, 
one should always remember that this option might lead to inconsistent calculation results. On the other hand, the TSK approach has a continuous output surface and offers better performance which makes it more suitable for real time (possibly selfadjusting) control applications.

In conclusion, we observe that MA or Larsen type fuzzy inference engines are more suitable for analytic applications where agent owners can express their existing knowledge without in depth mathematical knowledge whereas the TSK type fuzzy inference engine is capable of processing a larger amount of data and is thus more suitable for real time control applications.

\section{Usage Policies for Fuzzy Inference Methodologies}

In this section, we will discuss the suitability of the above discussed methodologies for the different tasks within our models. Also we will highlight a number of possible scenarios and analyze their impact on the performance of the models. Our previously introduced fuzzy models for the evaluation of trustworthiness and the measurement of service quality both have very different usage scenarios which need to be considered for the decision of the appropriate fuzzy inference method.

\section{Fuzzy Trust Evaluation Considerations}

The Fuzzy Trust Evaluation model needs to consider a potentially large number of opinions during the trust calculation process. Each of the delivered opinions will be fed into the fuzzy inference engine in order to receive a trustworthiness value for the Recommendation Queried Agent as perceived by the Recommending Agent. Furthermore, the Trusting Agent will evaluate the trustworthiness of several potential business partners during the service selection process. Since the Trusting Agent performs the trustworthiness evaluations before the business interaction with the final business partner, the performance of the fuzzy inference engine is a significant factor for the overall performance of the agent. Long delays caused by a slow performing trust evaluation model may lead to problems during the business interaction, especially in real time environments. The fuzzy rule base is unlikely to change frequently since it is defined once before the agent is deployed. Apart from minor adjustments the rule base will not be altered during future activities of the agent.

\section{Service Quality Review Considerations}

The service quality review process has a different purpose. This calculation process takes place after the business interaction took place and, thus, system performance is not of significant importance. Furthermore, the fuzzy based QoS Review inference engine will only be used once and not several hundred times as is the case during the trustworthiness evaluation process. However, the fuzzy rule base may be altered before each business interaction in order to reflect and comply with the individual service agreement between the service consumer (agent owner represented by the Trust- 
ing Agent) and the service provider (selected service represented by the Trusted Agent)

\section{Experimental Settings for Performance Tests}

In order to evaluate the performance of the MA type, the Larsen type, the TSK type, and the Singleton type fuzzy inference engines, we have set up a test environment using the Java-based jFuzzyLogic API [19]. We have designed the following fuzzy inference systems:

1. A Mamdani-Assilian type FIS

2. A Larsen type fFIS

3. A Takagi-Sugeno-Kang type FIS

4. A Singleton type FIS

All other settings are identical to ensure comparability. The underlying model for our FIS performance tests is based on our fuzzy trustworthiness model which has three input variables where two of the variables contain three fuzzy sets and one variable contains six fuzzy sets. The output fuzzy variable is composed of six fuzzy sets. All fuzzy sets are represented by overlapping Gaussian functions, except for the TSK output variable which is represented by six first order TSK functions and the Singleton output variable which is represented by six (singleton) values.

The output variable for the FIS is defined separately for the two methodologies. For the MA or Larsen type FIS we have defined one output fuzzy variable which contains three fuzzy sets. For the TSK type fuzzy inference engine we have represented the three linguistic terms (representing fuzzy sets) for the output variable as a first-order TSK function. For all modeled FIS we have chosen the centre of gravity defuzzification method as depicted in equation (1).

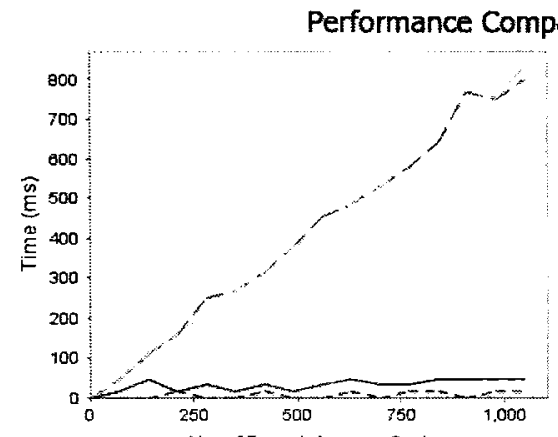

No. of Fuzzy Inference Cycles

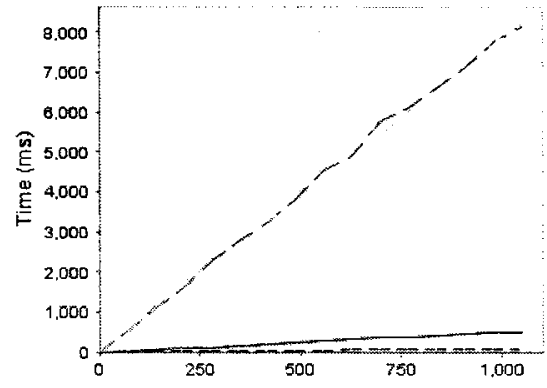

No. of Fuzzy Inference Cycles

- Takagi-Sugeno Mamdani - Larsen - - Singleton

Fig. 1. Performance Comparison of 4 FIS with a small rulebase and a large rulebase 
Our main goal was to measure the performance of the different types of fuzzy inference systems for a large number of cycles in the trust evaluation model. We have chosen to simulate the calculation of trustworthiness values for 15 potential business partners (Recommendation Queried Agents). We furthermore assume that neighboring agents (Recommending Agents) deliver 70 opinions in average for each Recommendation Queried Agent. Therefore, the FIS must process 1050 opinions overall.

\section{Policy Table}

Our simulation results show that the number of rules play a significant role for the performance of a FIS. The investigated FIS types exhibit a fast response time of time of $<1 \mathrm{~s}$ when only three fuzzy rules are defined. For a larger nulebase of 30 rules we observe expected longer response times for both systems. However, the TSK type FIS performs $\sim 16$ times faster compared to MA or Larsen type fuzzy inference engines. In all tests the Singleton type FIS exhibit the best performance, however this type of FIS was not considered during the design of the policy table. The limited modeling capabilities of Singleton type FIS result in more coarse grained trustworthiness and QoS values and thus affect the quality of our fuzzy models.

In situations where the tested fuzzy inference methodologies perform similar or where the response times are very high we favor the MA or Larsen type FIS because its setup requires less domain knowledge and allows consistent usage of linguistic terms rather then mathematical functions. The setup of a TSK type FIS requires the existence of experimental data or extensive domain knowledge.

Table 1. Policy Table for FIS methodologies

\begin{tabular}{|c|c|c|c|}
\hline $\begin{array}{c}\text { Fuzay } \\
\text { Rulebase }\end{array}$ & $\begin{array}{c}\text { \# of Inference } \\
\text { Cycles }\end{array}$ & $\begin{array}{c}\text { Trustworthiness Evaluation } \\
\text { Model }\end{array}$ & $\begin{array}{c}\text { Quality of Service Review } \\
\text { Model }\end{array}$ \\
\hline large & many & Takagi-Sugeno-Kang & Mamdani-Assilian or Larsen \\
\hline large & few & Mamdani-Assilian or Larsen & Mamdani-Assilian or Larsen \\
\hline small & many & Mamdani-Assilian or Larsen & Mamdani-Assilian or Larsen \\
\hline small & few & Mamdani-Assilian or Larsen & Mamdani-Assilian or Larsen \\
\hline
\end{tabular}

This analysis results in a policy table with which the agent will be equipped. This policy table supports the selection of an appropriate FIS for the different tasks the agent fulfills and the different situations the agent finds itself in.

\section{Conclusion}

Our analysis shows that Mamdani-Assilian (MA) or Larsen type and TakagiSugeno-Kang (TSK) type fuzzy inference methods have their merits in different 
situations. The Trusting Agent is equipped with a policy table to support dynamic decisions about which type of fuzzy inference system (FIS) it will select during the trustworthiness evaluation and quality of service (QoS) measurement process. Our tests show that in most situations the MA or Larsen type FIS is represents the preferred choice.

To measure the QoS after a business interaction, a MA or Larsen type FIS allows higher flexibility and a more intuitive approach for the agent owner during his initial system setup. The increased level of flexibility is given through a number of defuzzification methods from which the agent owner can choose, according to his personal preferences. Furthermore, a MA or Larsen type fuzzy inference system allows a linguistic modeling approach which offers a more intuitive approach in situations where extensive domain knowledge is absent. Also, since only a small set of data is processed during the QoS measurement within our fuzzy model, computational efficiency is not a crucial requirement.

In situations where the fuzzy rulebase is large and many inference cycles are required, the TSK type FIS is used due to its superior performance characteristics. The reduced computing efforts as a result of the more compact TSK type fuzzy inference approach for is especially suitable for the increased number of calculations in our fuzzy trust evaluation model. This high volume of calculations occurs if the agent operates in an information-rich environment where a large number of witness agents deliver their opinions about a potential business partner. The TSK type fuzzy inference approach significantly reduces the amount of computational power required to calculate overall trustworthiness values for all potential business partners in a timely manner and, thus, results in faster selection of business partners. We assume that in cases where the agent owner wants to increase the precision of the FIS by deploying a large rulebase, sufficient domain knowledge or previously recorded data already exists in order to design appropriate TSK type functions.

\section{References}

[1] S. Schmidt, R. Steele, T. Dillon, and E. Chang, "Building a Fuzzy Trust Network in Unsupervised Multi-agent Environments," in Lecture Notes in Computer Science, 2005, pp. 816-825.

[2] S. Schmidt, R. Steele, T. Dillon, and E. Chang, "Fuzzy Service Quality Review in Service Oriented Architectures," presented at IEEE International Conference on Fuzzy Systems, Vancouver, Canada, 2006.

[3] L. A. Zadeh, "Fuzzy sets," in Fuzzy sets, fuzzy logic, and fuzzy systems: selected papers by Lotfi A. Zadeh: World Scientific Publishing Co., Inc., 1996, pp. 1934.

[4] E. H. Mamdani and S. Assilian, "An experiment in linguistic synthesis with a fuzzy logic controller," Int. J. Man Machine Studies, vol. 7, pp. 1-13, 1975.

[5] T. Takagi and M. Sugeno, "Fuzzy identification of systems and its applications to modeling and control "IEEE Transactions on Systems, Man, and Cybernet$i c s$, vol. 15, pp. 116-132, 1985. 
[6] E. Chang, F. Hussain, and T. Dillon, Trust and Reputation for Service-oriented Environments, vol. 1: John Wiley \& Sons, 2006.

[7] R. Falcone, G. Pezzulo, and C. Castelfranchi, A Fuzzy Approach to a BeliefBased Trust Computation, 2003.

[8] B. Kosko, "Fuzzy Cognitive Maps," International Journal Man-Machine Studies, vol. 24, pp. 65-75, 1986.

[9] S. Jordi and S. Carles, "Reputation and social network analysis in multi-agent systems," in Proceedings of the first international joint conference on Autonomous agents and multiagent systems: part 1. Bologna, Italy: ACM Press, 2002.

[10] G. Chen, Z. Li, Z. Cheng, Z. Zhao, and H. Yan, A Fuzzy Trust Model for Multiagent System, 2005.

[11]Z. Shuqin, L. Dongxin, and Y. Yongtian, A Fuzzy Set Based Trust and Reputation Model in P2P Networks, 2004.

[12] C. Manara, P. Amato, A. Di Nola, M. Linawaty, and I. Pedaci, Software Implementation of Fuzzy Controller with Conditionally Firing Rules, and Experimental Comparisons, 2006.

[13] O. A. S. Youssef, "Applications of fuzzy inference mechanisms to power system relaying," 2004.

[14] P. M. Larsen, "Industrial Applications of Fuzzy Logic Control," International Journal of Man-Machine Studies, vol. 12, pp. 3-10, 1980.

[15] A. Konar, Computational Intelligence: Principles, Techniques and Applications. Heidelberg, Berlin: Springer, 2005.

[16] G. J. Klir and B. Yuan, Fuzzy sets and fuzzy logic: theory and applications. Upper Saddle River, N.J.: Prentice Hall, 1995.

[17] J. M. Mendel, Uncertain Rule-Based Fuzzy Logic Systems: Introduction and New Directions, 1 ed: Prentice Hall, 2001.

[18] M. Sugeno, "On stability of fuzzy systems expressed by fuzzy rules with singleton consequents," Fuzzy Systems, IEEE Transactions on, vol. 7, pp. 201-224, 1999.

[19] jFuzzyLogic: Open Source Fuzzy Logic (Java), http://juzzylogic.sourceforge.net 